\title{
Niveles de metales en partículas atmosféricas en la zona minera de carbón, norte de Colombia
}

\section{Metal levels in atmospheric particles in the coal mining zone, northern Colombia}

\author{
Carlos Doria-Argumedo \\ M. Sc. Química \\ Universidad de La Guajira \\ Riohacha, Colombia \\ cdoria@uniguajira.edu.co
}

\author{
Juan Fagundo-Castillo \\ Ph. D. Ciencias Químicas \\ Universidad de La Habana \\ La Habana, Cuba \\ Juan_fagundo@quimica.uh.cu
}

\begin{abstract}
Resumen- Dada la importancia del efecto sobre la salud de las partículas inhalables $\mathbf{P M}_{10}$ y los metales asociados a ellas, se evaluaron los niveles de algunos metales (Al, Cr, V, Hg, Pb, Ni, Zn, Cr, As, Se, Cd, Cu, Fe y Co) generados principalmente por las actividades mineras de carbón mineral en la zona de La Guajira, norte de Colombia. Todas las muestras fueron sometidas a un proceso de digestión ácida y analizadas por medio de espectrometría de masas con fuente de plasma de acoplamiento inductivo y la técnica de absorción atómica acoplada a vapor frío para el caso del $\mathrm{Hg}$. Con excepción del $\mathrm{Hg}$, todos los metales analizados presentaron diferencias estadísticamente significativas entre las zonas de muestreo. La gran mayoría de los metales se presentan en mayor concentración en la zona minera, en comparación con el sitio tomado como testigo ubicado en la ciudad de Riohacha, a una distancia aproximada de $150 \mathrm{~km}$. Por otra parte, para el período global los metales se presentan en mayor concentración en la época de sequía que en la de lluvias. El $\mathrm{Pb}, \mathrm{V}$ y $\mathrm{Hg}$ no exceden el estándar de calidad del aire establecido para Colombia, pero por su parte el As sobrepasa el límite permisible de la OMS en las zonas durante todo el período de muestreo. Adicionalmente el análisis estadístico permitió confirmar la actividad minera como la fuente principal de emisión de este tipo de contaminantes metálicos en la atmósfera sobre la región de La Guajira.
\end{abstract}

Palabras clave- Partículas atmosféricas, metales, zona minera, Colombia.

Abstract- Given the importance of the health effects of inhalable particles $\mathbf{P M}_{10}$ and metals associated with them , the levels were evaluated some metals ( $\mathrm{Al}, \mathrm{Cr}$, $\mathrm{V}$, $\mathrm{Hg}, \mathrm{Pb}, \mathrm{Ni}, \mathrm{Zn}, \mathrm{Cr}$, As , Se, cd , Cu, Fe and Co) generated mainly by coal mining activities in the area of La Guajira , northern Colombia. All samples were subjected to a process of acid digestion and analyzed by mass spectrometry source inductively coupled plasma and the atomic absorption technique coupled with cold steam for the case of $\mathrm{Hg}$. With the exception of $\mathrm{Hg}$, all metals analyzed showed statistically significant differences between sampling areas. The vast majority of the metals are present in higher concentrations in the mining area , compared with the site taken as a witness located in the city of Riohacha, at a distance of $150 \mathrm{Km}$. Moreover, for the overall period metals present in higher concentrations in the dry season than in the rainy. $\mathrm{Pb}, \mathrm{V}$ and $\mathrm{Hg}$ do not exceed the air quality standard set for Colombia, but meanwhile the Ace exceeds the permissible limit of OMS in areas throughout the sampling period. Further statistical analysis confirmed the mining activity as the main source of emission of this type of metal contaminants in the atmosphere over the region of La Guajira.

Keywords- Atmospheric particles, metals, mining area, Colombia

\section{INTRODUCCIÓN}

La calidad de un medio atmosférico viene determinada por el tipo de componentes que lo forman y el nivel de concentración en el que se encuentran en el aire [1]. Entre los indicadores más importantes de la contaminación atmosférica están el ozono, los hidrocarburos, los óxidos de nitrógeno, el monóxido de carbono y las partículas suspendidas [2]. Estas últimas son especialmente importantes debido a los efectos tóxicos que causan en el ser humano [3]. El diámetro de una partícula determina que sea o no "respirable". Se considera que las partículas menores de $10 \mu \mathrm{m}$ pueden ser inhaladas (esta fracción del aerosol atmosférico se designa como $\mathrm{PM}_{10}$ fracción inhalable) y que aquellas de diámetro inferior a 3,5 $\mu \mathrm{m}$ son 
respirables. El tamaño de la partícula determina, también, en qué lugar del tracto traqueo-bronquial quedará retenida y su peligrosidad relativa. Las partículas más pequeñas se depositan en los alvéolos pulmonares, mientras que las de diámetro en torno a los $15 \mu \mathrm{m}$ o mayores son retenidas en las fosas nasales [4]. Las partículas suspendidas forman una mezcla compleja de materiales sólidos y líquidos suspendidos en el aire, que pueden variar significativamente en tamaño, forma y composición, dependiendo fundamentalmente de su origen [5]. Las partículas atmosféricas están constituidas principalmente por sulfatos, nitratos, iones de amonio, ácidos, cloruros, agua, metales y carbono elemental y orgánico [6]. La mayoría de los estudios apuntan que el mayor impacto en la salud viene causado por las partículas de carbono elemental (CE), compuestos orgánicos (CO), especialmente hidrocarburos aromáticos policíclicos (HAP), sulfatos y nitratos, y determinados metales como As, Cd, Fe, Zn, Cr, Cu, Al, V, Ni y Pb [7]. Las emisiones de metales a la atmósfera se asocian con fuentes naturales (p.ej., tormentas de arena e incendios forestales) y fuentes antropogénicas (fijas y móviles) [8]. La resuspensión de partículas del suelo ha sido señalada como fuente principal de K, Mg y Mn presentes en polvo urbano de sedimento. Junto con la combustión de carbón, debe aportar también la mayor parte del Al, Ca, Ce, Cr, $\mathrm{Fe}$, La, Sc, Sr, Ti y Th [9]. El agente de resuspensión más importante es el viento, si bien no es el único. El tráfico de vehículos puede provocar la resuspensión de partículas, tanto por la acción mecánica de los neumáticos, como por la turbulencia inducida a su paso. Otros mecanismos son el tráfico peatonal y las actividades agrícolas y de construcción [10]. La incineración de residuos constituye la fuente principal de $\mathrm{Zn}, \mathrm{Cd}$ y Sb en atmósferas urbanas afectadas por esta actividad [11]. El Pb contenido en las partículas de polvo sedimentables proviene mayoritariamente de las emisiones de vehículos que utilizan gasolina como aditivo antidetonante. Por otra parte, se considera que los metales derivados de la emisión de los vehículos son principalmente $\mathrm{Cu}, \mathrm{Zn}, \mathrm{Cd}, \mathrm{Sb}, \mathrm{Ba}$ y $\mathrm{Pb}[12]$.

La minería de carbón a cielo abierto genera mayores impactos al medio ambiente que la minería subterránea. En particular, causa deterioro a la calidad del aire debido al material particulado y a los contaminantes gaseosos emitidos a la atmósfera [13]. No solo afecta el interior de las minas, sino también a las áreas de influencia externas. En la minería a cielo abierto, una gran cantidad de estéril es removido para destapar los depósitos minerales. Esto requiere excavadoras, vehículos de transporte, cargadores, bandas transportadoras, etc. Usualmente la explotación de carbón a cielo abierto involucra las siguientes actividades generales: 1. manejo de suelos (descapote, transporte y almacenamiento). 2. Perforación y voladura de material estéril. 3. Manejo de estéril (cargue, transporte y descarga) del estéril a zona de botaderos. 4. Manejo de carbón (cargue, transporte y descargue de carbón a zona de acopio) y 5 . Otras operaciones (erosión eólica, mantenimiento de vías y tráfico de vehículos) [14]. En el aspecto económico e industrial de La Guajira colombiana, la minería del carbón es la actividad más representativa en cuanto a extensión (69000 hectáreas) y explotación del recurso (30,2 millones de toneladas/año), la explotación es a cielo abierto y es una de las 10 mejores y más grandes empresas de Colombia, la compañía minera más grande del país, y unas de las minas de cielo abierto más grandes del mundo. Este grado importante de desarrollo económico e industrial minero en La Guajira, trae como consecuencia el aumento de los niveles de contaminación y deterioro de la calidad del aire, además, esta zona cuenta con un clima desértico y condiciones meteorológicas propicias para las altas concentraciones de polvo atmosférico. Por tal motivo, en esta investigación se evaluaron las concentraciones de algunos metales típicos (Al, Cd, V, Cr, Pb, Hg, As, Se, Bi, Fe, Co, Cu, $\mathrm{Ni}, \mathrm{Zn}$ ) generados por este tipo de fuente antrópica, presentes en el material particulado inhalable $\left(\mathrm{PM}_{10}\right)$ en las zonas de influencia del territorio guajiro. La determinación del contenido metálico en el polvo atmosférico de esta región es esencial para poder asociar las actividades mineras del carbón realizadas y su impacto ambiental.

\section{DESARROLLO EXPERIMENTAL}

\subsection{Muestreos}

Para determinar la composición metálica se colectaron partículas $\mathrm{PM}_{10}$ en los tres municipios del departamento de La Guajira (Colombia) que corresponden a la zona de influencia de la mina de 
carbón, Fig. 1. La Guajira se encuentra localizada entre los 10 grados 23 minutos y 12 grados 28 minutos de Latitud Norte y los 71 grados 06 minutos y 73 grados 39 minutos de longitud oeste del Greenwich; de marzo a diciembre de 2013. El monitoreo fue realizado teniendo en cuenta lo establecido en el protocolo y seguimiento de la calidad del aire [15]. Para la ubicación de las estaciones de muestreo se consideraron nueve puntos ubicados estratégicamente: Hatonuevo (1 estación), Albania (2 estaciones) Barrancas (6 estaciones) Fig. 2. Adicionalmente se tomó un punto testigo correspondiente al campus de la Universidad de La Guajira, que se encuentra a $5 \mathrm{~km}$ de la ciudad de Riohacha, y a una distancia de aproximadamente $150 \mathrm{~km}$ de la zona minera, cuya atmósfera se ve influenciada por el aerosol marino desde el punto de vista natural y el tráfico vehicular como influencia antropogénica.

Fig. 1. UBICACIÓN DE LA ZONA MINERA DEL CARBÓN MINERAL EN LA GUAJIRA. COLOMBIA

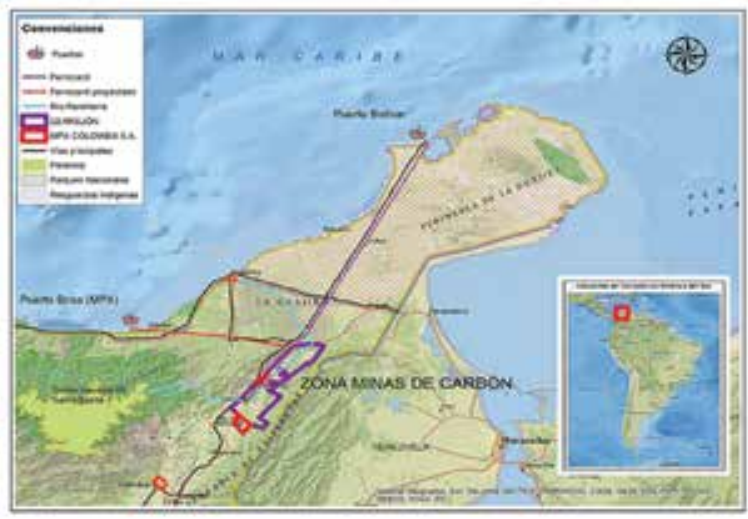

Fuente: Corporación Autónoma Regional de La Guajira - Corpoguajira.

Fig. 2. UBICACIÓN DE SITIOS DE MONITOREO DE PARTÍCULAS ATMOSFÉRICAS. LA GUAJIRA. COLOMBIA

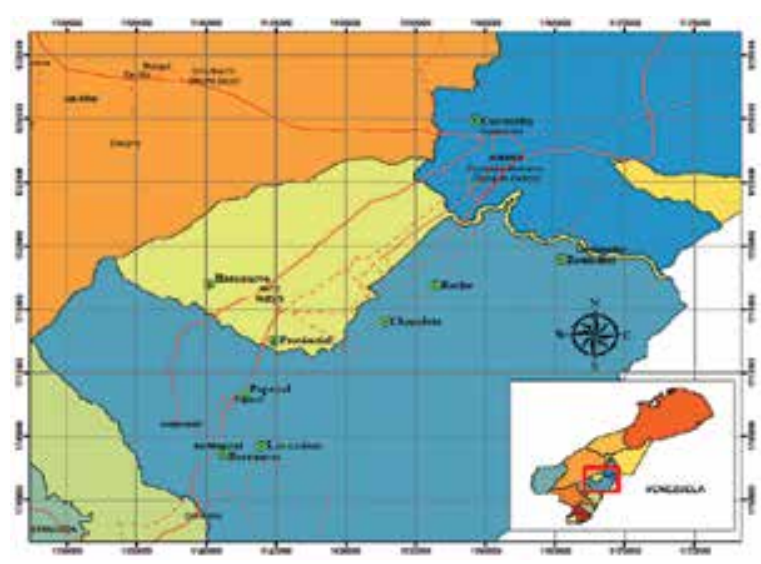

Fuente: autores.
Se recolectaron 100 muestras del polvo atmosférico por medio de filtros de cuarzo de $23,3 \mathrm{x}$ $25,4 \mathrm{~cm}$ mediante muestreadores de alto volumen $\mathrm{PM}_{10}$ (Hi-Vol VFC). Estos filtros cuentan con un $\mathrm{pH}$ ligeramente mayor a 7,5 y una eficiencia de colección del $99 \%$ de partículas de $>0.3 \mathrm{mi}-$ crómetros de diámetro [16]. Los filtros fueron acondicionados 24 horas antes de su pesaje para minimizar los errores de peso, a una temperatura de $25^{\circ} \mathrm{C}$ y una humedad relativa del $45 \%$. El tiempo de recolección fue de 24 horas continuas y una capacidad de flujo de aire entre 1,10 y $1,34 \mathrm{~m}^{3} / \mathrm{min}$.

\subsection{Extracción y análisis}

Después de colectadas las muestras de $\mathrm{PM}_{10}$, del acondicionamiento de los filtros y determinada la concentración de partículas mediante el método gravimétrico, se realizó la digestión de los filtros. Para tal fin a $1 / 4$ del filtro se le hizo una extracción ácida con $40 \mathrm{ml}$ de agua regia (65 $\mathrm{mL}$ de $\mathrm{HNO}_{3}+182 \mathrm{~mL}$ de $\mathrm{HCl}$, a un volumen total de $500 \mathrm{~mL}$ ) y $3 \mathrm{~mL}$ de $\mathrm{HClO}_{4}$ en vaso de precipitado. Para el $\mathrm{Hg} 1 / 4$ del filtro se digirió mediante la adición de una mezcla de ácidos: ácido sulfúrico $\left(\mathrm{H}_{2} \mathrm{SO}_{4}\right)$, ácido nítrico $\left(\mathrm{HNO}_{3}\right)$ y permanganato de potasio $\left(\mathrm{KMnO}_{4}\right)$. Pasadas las 24 horas, el contenido del vaso de precipitado se sometió a calentamiento (plancha de calentamiento) por una hora de 60 a $80^{\circ} \mathrm{C}$ hasta quedar un pequeño extracto, se agregó aproximadamente $20 \mathrm{ml}$ de agua desionizada recién hervida, se agitó y se filtró al vacío (filtro Whatman de fibra de vidrio $0,45 \mu \mathrm{m}, 0,47 \mathrm{~mm} \varnothing)$. El filtrado final se aforó a $250 \mathrm{~mL}$. Se refrigeró a $4^{\circ} \mathrm{C}$ hasta su posterior análisis [17]. Los metales $\mathrm{Co}, \mathrm{Cu}, \mathrm{Zn}, \mathrm{Cd}, \mathrm{Ni}, \mathrm{Bi}, \mathrm{Pb}, \mathrm{Al}, \mathrm{Cr}$, Se, As, V, y Fe fueron analizados de Espectrometría de Masas con fuente de Plasma de Acoplamiento Inductivo por medio de equipo ICP-MS Agilent Technologies 7000 series, utilizando Argón 5,0 como gas de arrastre a $0.9 \mathrm{~L} / \mathrm{min}$, bomba de nebulización a 0,4 rps, tiempo de retención de 0,3 s. y la utilización de solución patrón multielemental ICP: Solutions Plus Inc. ( $5 \% \mathrm{HNO}_{3}$ ). El Hg fue analizado mediante la técnica de Absorción Atómica Acoplada a Vapor Frío por medio del equipo ICE 3500 Thermo Scientifc con corrección de fondo Zeeman y lámpara de deuterio $D_{2}$. 


\subsection{Tratamiento estadístico}

Para el análisis estadístico se aplicaron varios criterios como la prueba de rechazo de Chauvenet, pruebas de medias normales con varianza y medias desconocidas t-Student, coeficiente de correlación lineal, series de tiempo (para el caso de las concentraciones en los diferentes períodos de monitoreo), análisis de correlación (entre concentración de iones) y análisis de componentes principales para las concentraciones en los diferentes sitios de monitoreo con el objeto de determinar posibles asociaciones (procedimiento de rotación Varimax); mediante la utilización del software SPSS versión 21 IBM. Teniendo en cuenta que durante los procesos de extracción y análisis de las muestras se pierde cantidad de masa de cada ión, se procedió a determinar los respectivos porcentajes de recuperación en muestra preparada de concentración conocida y el respectivo análisis de un filtro de cuarzo en blanco.

\subsection{Estandarización del método analítico}

Para la validación del método y el tratamiento de las muestras se tuvo en cuenta la evaluación de los parámetros de linealidad, precisión, exactitud, límite de detección y de cuantificación. Para la linealidad, se tomaron alícuotas correspondientes a seis concentraciones diferentes $(0,0005,0,001$, $0,005,0,01,0,1$ y $0,5 \mathrm{mg} / \mathrm{L}$ ) de las disoluciones patrón en el rango de aplicación del método, la precisión fue realizada por determinación de la desviación estándar y coeficiente de variación (CV) de los resultados del análisis, para $n=10$, de muestras reales en condiciones de repetibilidad, la exactitud fue definida en términos de porcentaje de error relativo, para los estándares analizados este porcentaje de error relativo fue menor a lo esperado $(<5 \%)$ para dos analistas diferentes. La evaluación de los parámetros de calidad fue realizada para la concentración de 0,1 mg/L. Para la determinación de límite de detección y de cuantificación se realizaron determinaciones en muestras de blancos o concentración baja del analito para $n=10$.

\section{RESULTADOS}

\subsection{Control de calidad}

En la tabla I se muestran los resultados de los criterios tenidos en cuenta para la validación de método analítico. En general los valores registrados para este estudio indicaron condiciones aceptables, garantizando la confiabilidad de los resultados.

\subsection{Condiciones meteorológicas durante el período de muestreo}

En La Guajira la dispersión de las partículas suspendidas y su disolución en la atmósfera está condicionada por los factores climáticos característicos de una zona insular que presenta temperatura promedio de $29,3^{\circ} \mathrm{C}$ (con intervalo $22,5^{\circ} \mathrm{C}$ - $34,7^{\circ} \mathrm{C}$ ), humedad relativa de $70,5 \%$ (con intervalo $34 \%$ - 90\%), velocidad del viento de $6,8 \mathrm{~km} / \mathrm{h}$ (con intervalo de $0,4 \mathrm{~km} / \mathrm{h}-10,9 \mathrm{~km} / \mathrm{h}$ ) en las direcciones principalmente oeste-noroeste y noroeste, Fig. 3, y promedio de lluvia de $2,4 \mathrm{~mm}$ (con intervalo de 0,25 mm - 38,10 mm) [18].

\subsection{Concentración de partículas y metales}

En la zona minera del carbón, la resuspensión de partículas se ve favorecida por las actividades de excavación y el tránsito vehicular que presenta, lo que explica que se muestren concentraciones anuales entre los 30,0 y $40,0 \mu \mathrm{g} / \mathrm{m}^{3}$, tabla II; observándose que estos valores no superan el límite máximo permitido de $90 \mathrm{\mu g} / \mathrm{m}^{3}$ propuesto por la OMS o de $50 \mu \mathrm{g} / \mathrm{m}^{3}$ de acuerdo con la Norma Oficial colombiana [19].

Las concentraciones más elevadas de metales en partículas atmosféricas $\mathrm{PM}_{10}$ se registraron para el $\mathrm{Al}\left(=0,67 \mu \mathrm{g} / \mathrm{m}^{3}\right)$ y las más bajas para el $\mathrm{Pb}\left(=0,008 \mu \mathrm{g} / \mathrm{m}^{3}\right)$, tabla II. Las concentraciones de Al, V, Cr, Fe, Se y As tendieron a aumentar durante los meses de mayo y junio, adicionalmente, las concentraciones de estos metales tendieron a disminuir durante los meses de octubre y noviembre. A partir de lo anterior, los resultados indicaron que hubo un aumento en las concentraciones de los elementos metálicos en estudio en tiempo de sequía y una disminución en época de Iluvia, Fig. 4. El aumento en la concentración durante el verano puede proponerse que se debe a factores como la resuspensión de partículas al suelo y el transporte de aerosoles marinos influenciados por los vientos de mayor intensidad, con predominancia en la dirección NNE y que durante la época de invierno el lavado de las partículas 
solubles disminuye su concentración atmosférica total [20].

El Hg y Bi presentaron concentraciones constantes en los diferentes tiempos. El Pb, por su parte, solo se detectó concentración durante los meses de junio y julio. Los elementos que presentaron concentraciones menores al límite de detección fueron $\mathrm{Co}, \mathrm{Cu}, \mathrm{Zn}, \mathrm{Cd}$ y $\mathrm{Ni}$.

Al comparar las concentraciones de los metales con respecto a la legislación colombiana [19] y los recomendados por la OMS, se puede observar que las concentraciones de $\mathrm{Pb}, \mathrm{V}$ y $\mathrm{Hg}$ no superaron los límites permisibles. Pero el As se encuentra muy por encima de lo permitido por la OMS, Fig. 5.

Las concentraciones de $\mathrm{Fe} \mathrm{y} \mathrm{Pb}$ se encuentran muy por debajo de las registradas en la zona industrial de Puente Aranda de Bogotá y Pamplona en Colombia [6], [21] y de la ciudad de Potosí (Mé- xico) [22]. El Cr se presenta en concentración muy por encima de lo registrado en zonas de alto tráfico vehicular del estado Zulia (Venezuela), [23], tabla III.

La especie química de $\mathrm{Pb}$ más abundante en el polvo atmosférico urbano es el $\mathrm{PbSO}_{4}$. Otras especies mayoritarias son el $\mathrm{PbO}$ y $\mathrm{Pb}_{3} \mathrm{O}_{4}$. Principalmente el $\mathrm{PbSO}_{4}$ está presente en atmósferas urbanas contaminadas por emisiones de vehículos, y proceden de la relación del $\mathrm{PbBrCl}$ con sulfatos de carácter ácido y neutro, respectivamente [24]. $\mathrm{El} \mathrm{Pb}$ también se encuentra como componente mayoritario en muestras tomadas en aparcamientos, gasolineras, etc.... es decir, en lugares donde los motores de los vehículos arrancan en frío, y su origen está, muy probablemente, en el proceso de deposición en el sistema de escape que se produce en tales situaciones [25]. Entre un $60 \%$ y un $75 \%$ del $\mathrm{Pb}$ añadido a las gasolinas es emitido por el sistema de escape de los vehículos como sales inorgánicas de $\mathrm{Pb}$ y alrededor del $1 \%$ es emitido como $\mathrm{Pb}$ tetralquilo [26].

TABLA I

RESULTADOS DE LOS PARÁMETROS DE CONTROL DE CALIDAD DEL MÉTODO ANALÍTICO CORRESPONDIENTE A LOS METALES

\begin{tabular}{|c|c|c|c|c|c|c|}
\hline Metales & $\mathbf{R}$ & Desviación estándar & Coeficiente de variación (\%) & Exactitud (\%) & LDM $=\mathbf{X + t *}$ & LCM=X+3*s \\
\hline $\mathrm{Al}$ & 0,99957 & 0,04 & 0.055 & 4,08 & 0,0008 & 0,0011 \\
\hline $\mathrm{Cr}$ & 0,99937 & 0,05 & 0,057 & 4,09 & 0,0009 & 0,0012 \\
\hline $\mathrm{V}$ & 0,99998 & 0,06 & 0,042 & 4,18 & 0,0002 & 0,0006 \\
\hline $\mathrm{Fe}$ & 0,99936 & 0,03 & 0,051 & 4,15 & 0,0026 & 0,029 \\
\hline $\mathrm{Se}$ & 0,99966 & 0,07 & 0,047 & 4,23 & 0,0012 & 0,0016 \\
\hline $\mathrm{As}$ & 0,99975 & 0,03 & 0,045 & 4,88 & 0,0011 & 0,0013 \\
\hline $\mathrm{Hg}$ & 0,99990 & 0,04 & 0,056 & 4,03 & 0,0009 & 0,0014 \\
\hline $\mathrm{Bi}$ & 0,99979 & 0,08 & 0,039 & 4,01 & 0,0032 & 0,0036 \\
\hline $\mathrm{Pb}$ & 0,99983 & 0,05 & 0,045 & 4,35 & 0,0030 & 0,0033 \\
\hline
\end{tabular}

Fuente: Los autores

TABLA II

CONCENTRACIÓN ANUAL DE PM ${ }_{10}$ Y DEL CONTENIDO METÁLICO $\left(\mu \mathrm{g} / \mathrm{m}^{3}\right)$

\begin{tabular}{|l|c|c|c|c|c|c|c|c|c|c|c|}
\hline \multicolumn{1}{|c|}{ Sitio } & No de observaciones & Conc & Al & V & Cr & Fe & As & Se & Hg & $\mathbf{B i}$ & $\mathbf{P b}$ \\
\hline Universidad & 10 & 0,28 & 0,31 & 0,33 & 0,40 & 0,30 & 0,14 & 0,70 & 0,035 & 0,31 & 0,00 \\
\hline Hatonuevo & 10 & 53,1 & 1,07 & 0,35 & 0,45 & 0,37 & 0,14 & 0,081 & 0,035 & 0,31 & 0,00 \\
\hline Albania & 10 & 30,2 & 0,89 & 0,36 & 0,48 & 0,46 & 0,14 & 0,091 & 0,035 & 0,31 & 0,006 \\
\hline Barrancas & 10 & 33,6 & 0,74 & 0,37 & 0,47 & 0,46 & 0,15 & 0,055 & 0,035 & 0,31 & 0,02 \\
\hline Promedio & 10 & 42,01 & 0,66 & 0,36 & 0,47 & 0,40 & 0,14 & 0,082 & 0,035 & 0,31 & 0,008 \\
\hline
\end{tabular}

Fuente: Los autores 
TABLA III

COMPARACIÓN DE CONCENTRACIONES $\left(\mu \mathrm{g} / \mathrm{m}^{3}\right)$ DE METALES EN PARTÍCULAS ATMOSFÉRICAS DE LA ZONA MINERA DE CARBÓN DE LA GUAJIRA CON OTRAS ZONAS DE COLOMBIA Y DEL MUNDO

\begin{tabular}{|l|c|c|c|c|}
\hline \multicolumn{1}{|c|}{ Lugar } & Cr & Pb & Fe & As \\
\hline $\begin{array}{l}\text { La Guajira } \\
\text { Este estudio }\end{array}$ & 0,47 & 0,009 & 0,42 & 0,15 \\
\hline Bogotá (Colombia) [6] & 0,25 & 1,50 & 2,12 & \\
\hline Pamplona (Colombia)[21] & 0,88 & 1,02 & 22,97 & \\
\hline Potosí (México)[22] & & 0,26 & 6,79 & 0,26 \\
\hline Edo Zulia (Venezuela) [23] & 0,05 & 1,13 & & \\
\hline
\end{tabular}

Fuente: Los autores

Fig. 3. ROSA DE LOS VIENTOS DE LA ZONA DE ESTUDIO. Año 2013

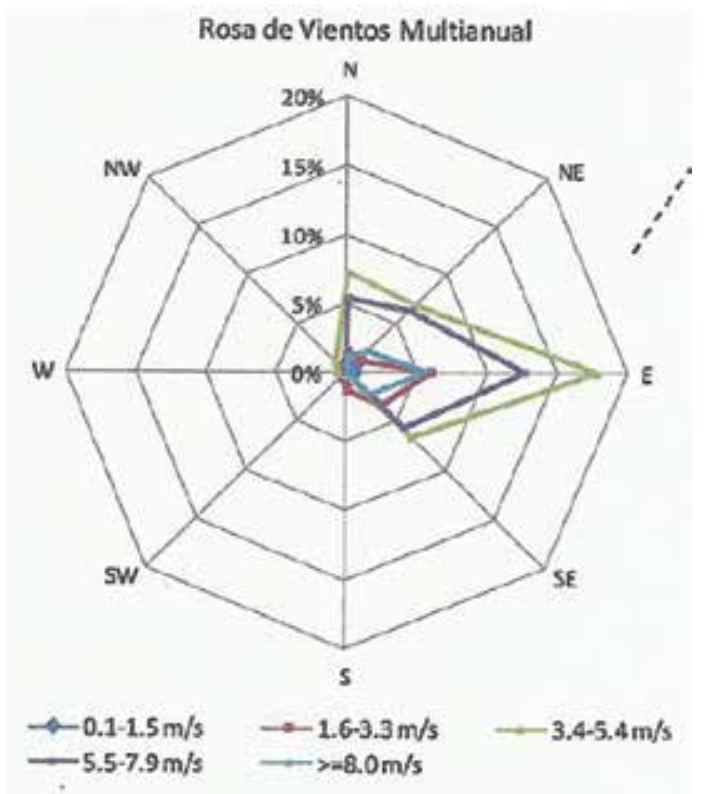

Fuente: Corporación Autónoma Regional de La Guajira-Corpoguajira

Fig. 4. COMPARACIÓN DE LAS CONCENTRACIONES DE ALGUNOS METALES EN PARTÍCULAS ATMOSFÉRICAS CON ESTÁNDARES DE CALIDAD DEL AIRE

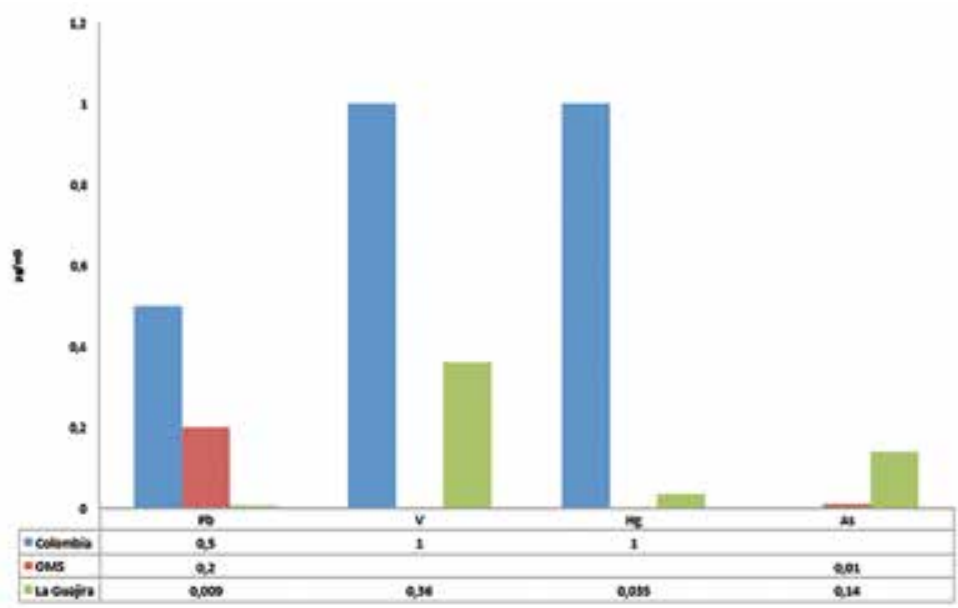

Fuente: Los autores. 
Fig. 5. DIFERENCIA DE LAS CONCENTRACIONES DE LOS METALES EN LAS PM ${ }_{10}$ EN LAS ÉPOCAS CLIMÁTICAS DE VERANO E INVIERNO
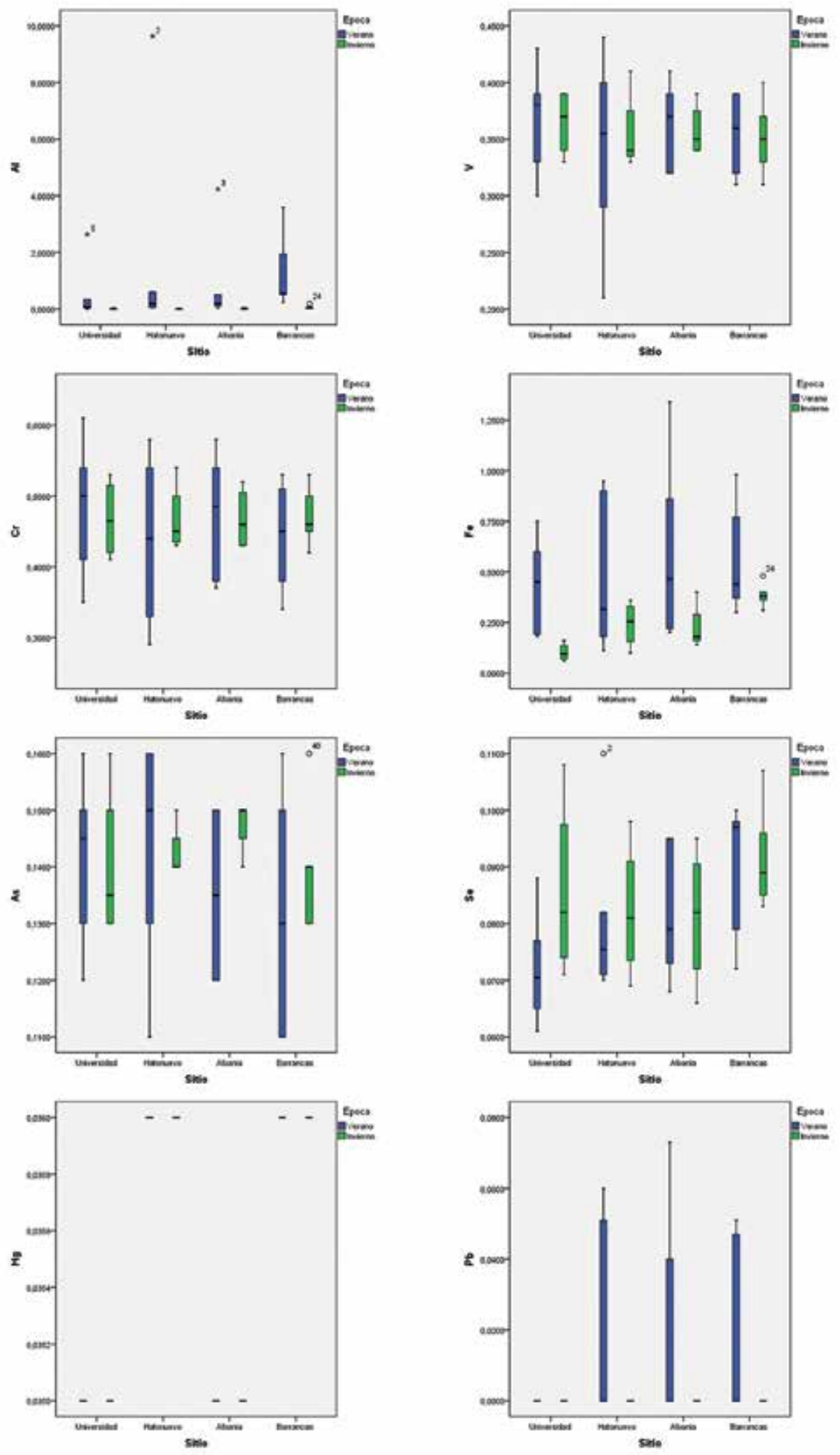

Fuente: Los autores. 
La presentación de los datos de $\mathrm{V}$ y $\mathrm{Cr}$ viene dada por una correlación significativa $(0,946)$, al igual que los de As y Se $(0,455)$ y Al y Fe $(0,479)$ tabla IV. Se puede decir que la presencia de los metales $\mathrm{V}, \mathrm{Cr}, \mathrm{Al}$ y Se en las partículas tienen una mayor relación entre sí, debido a que pueden estar asociados a una misma fuente de origen. Para el caso de los demás metales se observa que no se comportan de la misma manera en un sitio y otro, es decir, presentan máximos en distintos puntos y en diferentes tiempos, dando a indicar que el origen de estos metales en el aire no corresponde a los mismos focos de emisión. La resuspensión de partículas de suelo junto con la combustión del carbón se consideran como los principales aportantes de Cr, Fe y Al a la atmósfera [9].

El Al se utiliza como elemento indicador de partículas procedentes de la combustión del carbón y/o de la resuspensión de suelo por su composición aluminio-silicatada. El Se tiene relación con partículas procedentes de la combustión del carbón mayoritariamente que las producidas por la resuspensión del suelo [27]. El V se ha utilizado como indicador de combustión de crudos de petróleo y gasoil en la composición química de partículas aerotransportadas. El petróleo crudo también se considera como fuente de elementos en concentraciones menores como el Al, Fe, Zn, Cu y $\mathrm{Pb}$ [28]. Algunos autores también han atribuido la presencia de $\mathrm{V}$ a la resuspensión de partículas de suelo aerotransportadas desde zonas árido-desérticas [29].

\subsection{Distribución espacial de los metales en $\mathrm{PM}_{10}$}

Como se observa en la Fig. 6 los metales aparecen con mayor concentración en la zona minera que en la universidad de La Guajira, tomada como testigo, a excepción del Cr y V, hecho que nos indica la posible influencia de las actividades mineras del carbón en el contenido metálico del polvo atmosférico de esta zona del país y de la influencia del tráfico automotor para el caso de Riohacha. Con excepción del $\mathrm{Hg}$ (significancia $=0,0$ ), todos los metales analizados presentaron diferencias estadísticamente significativas entre las zonas de muestreo.

En términos generales, a excepción del Al, no se observa una variación significativa de las concen- traciones de los metales contenida en las partículas en cada sitio de estudio en los distintos tiempos tabla $\mathrm{V}$, indicando en principio que estas especies son producidas por una misma fuente de manera constante, la cual debe ser la actividad minera del carbón.

Con relación a la asociación de los metales, en la Fig. 7, que corresponde a los análisis de rotación indica que en el factor 1 las variables $\mathrm{Al}, \mathrm{Pb}, \mathrm{Fe}, \mathrm{V}$, $\mathrm{Se}, \mathrm{As}, \mathrm{Cr}$ estadísticamente conciernen a metales asociados, esto podría pertenecer a su relación basada en que estas especies provienen de la misma fuente antropogénica como lo es la actividad minera.

Las actividades de extracción y transporte del carbón demandan la utilización de combustibles como el fuel oíl y crudo, los cuales están relacionados con la emisión de $\mathrm{Pb}, \mathrm{Cr}$, Cu y $\mathrm{Zn}$ [30]. En un estudio realizado en la ciudad de Bogotá sobre exposición de emisiones en una zona de alto tráfico vehicular se reportaron concentraciones máximas de 1 y $0,6 \mu g / \mathrm{m}^{3}$ de $\mathrm{Pb}$ y $0,2 \mu \mathrm{g} / \mathrm{m}^{3}$ de $\mathrm{Cr}$, las cuales atribuye a la utilización de gasolina con alto contenido de plomo [31]. Para el caso del Fe este metal está asociado principalmente al polvo que se encuentra en la corteza terrestre y también a procesos de combustión con carbón, en menor medida, se relaciona con las emisiones vehiculares [32]. El hierro es uno de los metales que se encuentra naturalmente en el suelo, lo cual genera altas concentraciones del mismo en el aire [33].

\section{CONCLUSIONES}

Los niveles de $\mathrm{Pb}, \mathrm{V}$ y $\mathrm{Hg}$ reportados en este estudio en la zona minera al norte de Colombia, no superan el estándar anual nacional y los establecidos por la OMS, sin embargo, existen niveles de As muy por encima de lo permitido por esta norma internacional.

Se observa poca dispersión de los metales, es decir que no son aerotransportados, observándose concentraciones casi invariables en los tres sitios donde se encuentra el complejo carbonífero. La poca variabilidad de las concentraciones se mantiene en las épocas climáticas verano e invierno. 

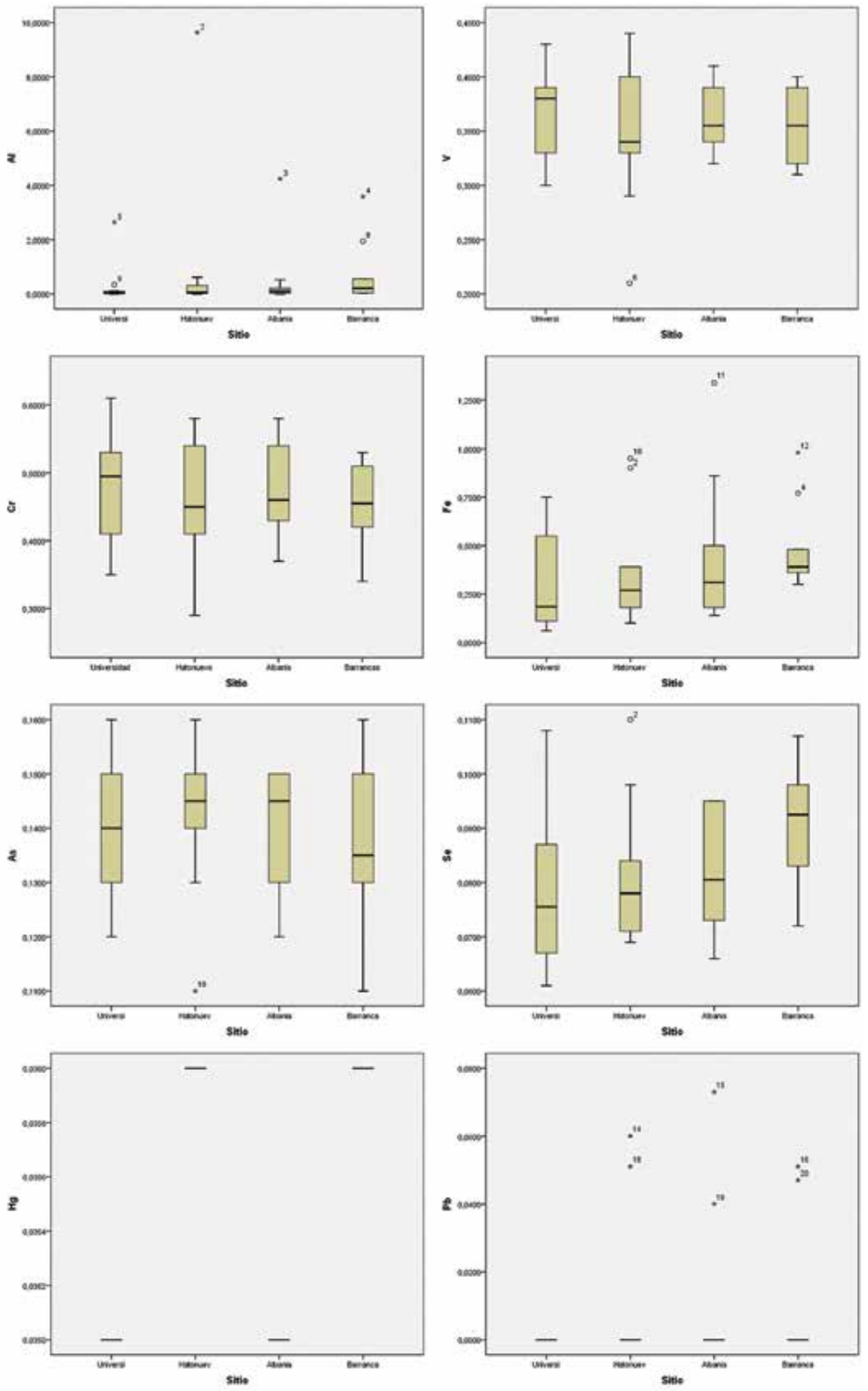

Fuente: Los autores. 
Todos los metales evaluados en las partículas atmosféricas son emitidos a la atmósfera producto de las actividades mineras, relacionadas con las excavaciones (Fe, Se, As) y el parque automotor (V, Pb, Hg, Cr).

Los niveles metálicos en la zona testigo se mostraron muy por debajo a los reportados en la zona minera del carbón, lo cual permite catalogar a Riohacha como una zona limpia y que constituye una buena estación blanco para estudios de concentraciones metálicas. La presencia de $\mathrm{Cr}$ y $\mathrm{Hg}$ en la atmósfera de Riohacha puede estar relacionada con el tráfico automotor, como principal fuente antrópica.

TABLA IV MATRIZ DE CORRELACIONES ENTRE LOS IONES METÁLICOS DE LAS PARTÍCULAS ATMOSFÉRICAS

\begin{tabular}{|c|c|c|c|c|c|c|c|c|}
\hline & $\mathrm{Al}$ & V & $\mathrm{Cr}$ & $\mathrm{Fe}$ & As & $\mathrm{Se}$ & $\mathrm{Hg}$ & $\mathrm{Pb}$ \\
\hline $\mathrm{Al}$ & 1 & $-0,169$ & $-0,279$ & 0,479 & $-0,190$ & 0,474 & 0,136 & $-0,99$ \\
\hline V & & 1 & 0,946 & 0,107 & 0,288 & $-0,311$ & $-0,162$ & 0,328 \\
\hline $\mathrm{Cr}$ & & & 1 & 0,116 & 0,364 & $-0,343$ & $-0,145$ & 0.228 \\
\hline $\mathrm{Fe}$ & & & & 1 & $-0,397$ & 0,299 & 0,091 & $-0,061$ \\
\hline As & & & & & 1 & $-0,455$ & $-0,035$ & 0,428 \\
\hline $\mathrm{Se}$ & & & & & & 1 & 0,257 & $-0,324$ \\
\hline $\mathrm{Hg}$ & & & & & & & 1 & 0,123 \\
\hline $\mathrm{Pb}$ & & & & & & & & 1 \\
\hline
\end{tabular}

Fuente: Los autores

TABLA V

VALORES DE LOS COEFICIENTES DE VARIACIÓN Y DESVIACIÓN DE LAS CONCENTRACIONES DE LOS METALES EN LOS SITIOS DE MUESTREO

\begin{tabular}{|l|c|c|c|c|}
\hline \multicolumn{1}{|c|}{ Metal } & Universidad & Hatonuevo & Albania & Barrancas \\
\hline $\mathrm{Al}$ & 2,55 & 2,81 & 2,43 & 1,61 \\
\hline $\mathrm{V}$ & 0,10 & 0.20 & 0,08 & 0,09 \\
\hline $\mathrm{Cr}$ & 0.16 & 0,18 & 0,15 & 0,13 \\
\hline $\mathrm{Fe}$ & 0,81 & 0,81 & 0,86 & 0,46 \\
\hline $\mathrm{As}$ & 0,09 & 0,09 & 0,09 & 0,13 \\
\hline $\mathrm{Se}$ & 0,17 & 0,16 & 0,13 & 0,11 \\
\hline $\mathrm{Hg}$ & $*$ & $*$ & $*$ & $*$ \\
\hline $\mathrm{Pb}$ & $*$ & 2,09 & 2,27 & 2,23 \\
\hline $\mathrm{Bi}$ & $*$ & $*$ & $*$ & $*$ \\
\hline
\end{tabular}

*Desviación estándar $=0,0$ 
Fig. 7. MATRIZ DE FACTORES ROTADOS CORRESPONDIENTE A LOS METALES EN PARTÍCULAS ATMOSFÉRICAS

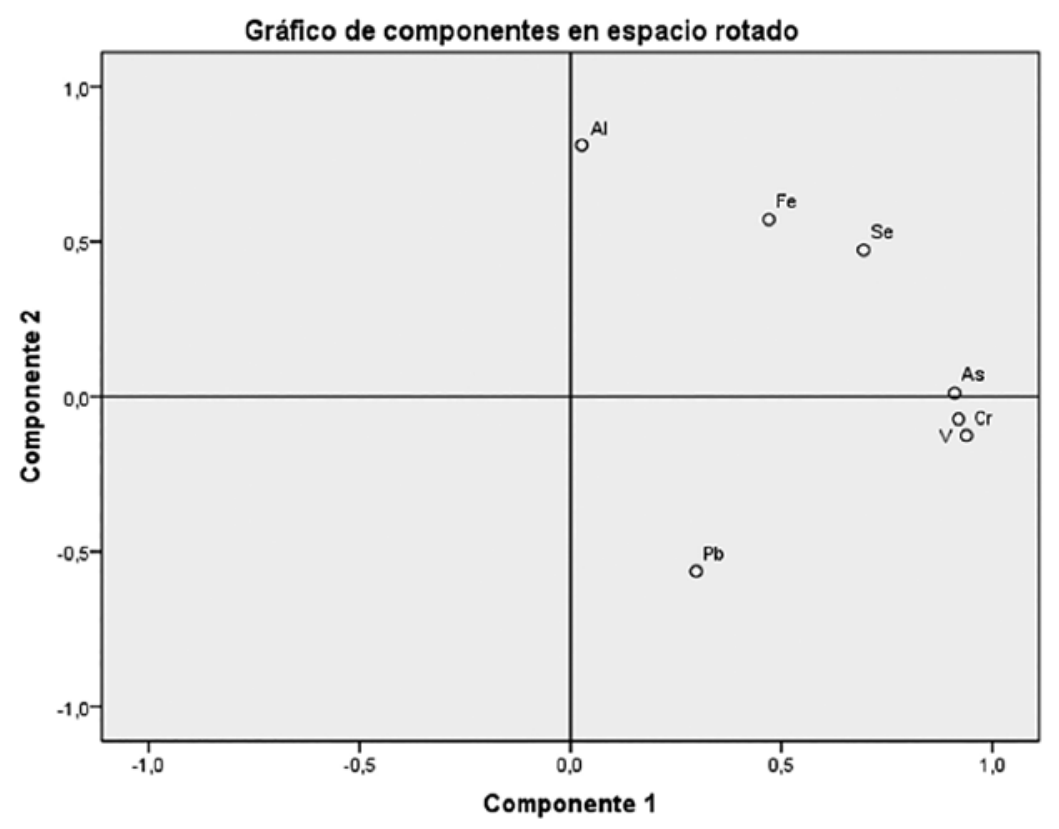

Fuente: Los autores.

\section{REFERENCIAS}

[1] X. Doménech, "Química Atmosférica. Origen y efectos de la contaminación". Miraguano Ed. Madrid, España, 1995.

[2] R. Dams, J. Billet, C. Block, M. Demuynck, M. Janssens, "Complete Chemical Analysis of airborne particulates", Atmos. Environ, vol 9, pp. 1099-1106, 1995.

[3] A. Choudhury, M. Gordian, S. Morris, "Associations between respiratory illness and PM10 air pollution", Arch. Environ. Health, vol 52, pp. 113-117, 1997.

[4] G. Sehmel, "Particle resuspension. A Review”, Environmental International, vol 4, pp. 107-127, 1980.

[5] A. Fernández, M. Ternero, F. Barragán, J. Jiménez, “An approach to characterization of sources of urban airborne particles through heavy metal speciation", Chemosphere - Global Change Science, vol 2, pp. 123136, 2000.

[6] L. Pérez, L. Hernández L., “Determinación de metales pesados en partículas respirables e identificación de fuentes de emisión, a partir de un muestreo atmosférico en la localidad de Puente Aranda en la ciudad de Bogotá". Tesis de Pregrado. Facultad de Ingeniería Ambiental y Sanitaria. Universidad de La Salle, Bogotá, 2006.
[7] WHO, "Health aspects of air pollution with particulate matter, ozone and nitrogen dioxide", World Health Organization, Ginebra, 2003.

[8] R. García. "Determinación de metales pesados en la precipitación pluvial de una zona urbana (Ciudad de México) y de una zona rural (Rancho Viejo, Edo. de México", Tesis de maestría. Universidad Nacional Autónoma de México. México D.F., 2007.

[9] C. Boni, E. Caruso, E. Cereda, G. Lombardo, G. Braga, P. Redaelli, "Particulate matter elemental characterization in urban areas. Pollution and source identification", Journal of Aerosol Science, vol 19, pp. 12711274, 1988.

[10] G. Kowalczyk, C. Choquette, "Identification of atmospheric particulate sources in Washington D.C. Using Chemical Elements Balances". Atmospheric Environmental Science and Technology, vol 16, pp. 79-90, 1982.

[11] G. Kowalczyk, C. Choquette, G. Gordon, "Chemical elements balances and identification of air pollution sources in Washington". Atmospheric and Environmental, vol 12, pp. 1143-1153, 1978.

[12] J. Sternbeck, A. Sjödin, K. Andreasso, "Metal emissions from road traffic and the influence of resuspension - results from two tunnel studies", Atmospheric Environmental, vol 36, pp. 4735-4744, 2002. 
[13] M. Ghose, “Opencast coal mining in India: Asssesig Air Pollutant Emission Environmental Quality Management", Autum, pp. 35-51, 2007.

[14] L. Angulo, J. Huertas, G. Restrepo, “Caracterización de Partículas Suspendidas (PST) y Partículas Respirables (PM10) producidas en Áreas de Explotación Carbonífera a Cielo Abierto", Información Tecnológica, vol 22, pp. 23-34, 2011.

[15] MAVDT- Ministerio de Medio Ambiente, Vivienda y Desarrollo Territorial, "Protocolo para el monitoreo y seguimiento de la calidad del aire", Bogotá, Colombia, 2008.

[16] REDAIRE. Red de Vigilancia de la Calidad del Aire, "Protocolo para muestreo de partículas respirables (PM10) utilizando el equipo muestreador de alto volumen PM10 de flujo constante", Disponible en http:/ www.unalmed.edu.co/redaire/. Acessado en julio de 2015.

[17] C. Doria, "Estudio sobre la composición química de la Iluvia y partículas suspendidas en un sitio del área de influencia de las actividades de explotación del carbón mineral en La Guajira colombiana", Tesis de maestría. Facultad Experimental de Ciencias. Universidad del Zulia, Maracaibo, 2002.

[18] Corpoguajira-Corporación Autónoma Regional de La Guajira, "Condiciones climáticas de La Guajira año 2013". División de Control y Monitoreo Ambiental, Reporte Anual. Riohacha, 2014.

[19] MAVDT-Ministerio de Ambiente, Vivienda y Desarrollo Territorial. Resolución 610 "Por la cual se modifica la Resolución 601 del 4 de abril de 2006" la Norma de Calidad del Aire o Nivel de Inmisión, para todo el territorio nacional, Bogotá, 2010.

[20] J. Galloway, G. Likans, W. Keene, J. Miller. "The composition in remore areas of the world", Journal Geophysic, vol 87, pp. 8771-8786, 1982.

[21] A. Quijano, M. Quijano, J. Henao, “Caracterización fisicoquímica del material particulado fracción respirable PM 2,5 en Pamplona Norte de Santander - Colombia", Bistua, vol 8 no 1, 2010.

[22] A. Aragón, A. Campos, R. Layva, M. Hernández, N. Miranda, K. Luszczewski, K., "Influencia de emisiones industriales en el polvo atmosférico de la ciudad de Potosí, México", Revista Internacional de Contaminación Ambiental, vol 22 no 1, pp. 0188-4999, 2006.
[23] A. Machado, N. García, C. García, L. Acosta, A. Córdova, M. Linares, D. Giraldoth, H. Velásquez, “Contaminación por metales ( $\mathrm{Pb}, \mathrm{Zn}, \mathrm{Ni}$ y $\mathrm{Cr}$ ) en aire, sedimentos viales y suelo en una zona de alto tráfico vehicular", Int. Contam. Ambient, vol 24 no 4, 2008.

[24] D. Biggins, R. Harrison, "Chemical speciation of lead compounds in street dusts", Enviromental Science and Technology, vol 14, pp. 336-339, 1980.

[25] K. Olson, R. Skogerboe R., "Identification of soil lead compounds from automovile sorces". Environmental Science and Technology, vol 9, pp. 227-230, 1975.

[26] M. García, "Geoquímica Urbana de elementos traza". Tesis Doctoral, Departamento de Ingeniería Química y Combustibles. Universidad Poitécnica de Madrid, Madrid, 1995.

[27] U. Tomza, "Trace elements in the atmospheric aerosol and Katowice, Poland", Technical report Instituut voor Nucleaire Wetenschappen, Rijksuniversiteit Gent. Bélgica, 1984

[28] M. Sadiq, A. Mian, "Nikel and Vanadium in air particulates at Dharahn (Saudi Arabia) during and after the Kuwait Oil Fires". Atmospheric and Environment, vol 28, pp 2249-2253, 1994.

[29] P. Cornille, W. Maenhaut, J. Pacyna. "Sources and Characteristics of the atmospheric aerosol near Damascus. Syria". Atmospheric and Environment, vol 24A, pp 1083-1093, 1990.

[30] EPA, "Compilation of Air Pollutants Emission Factors", AP-42, 5th ed., Volume I: Stationary Point and Area Sources. EE.UU., 2002.

[31] C. Cárdenas, "Estudio exploratorio de la exposición de trabajadores ambulantes a las emisiones vehiculares en inmediaciones de la Universidad Nacional de Colombia". Tesis de pregrado. Facultad de Ingeniería. Universidad Nacional de Colombia, Bogotá, Colombia, 2003.

[32] G. Fang, N. Cheng, W. Yuh-Shen, W. Vicky, P. Peter, Y. Ding-Guor, C. Shun-Chin, C. Chia-Chium C., "Characterization of particulate, metallic elements of TSP, PM2.5 and PM2.5-10 aerosols at a farm sampling site in Taiwan, Taichung", Institute of Technology, Sha-Lu, Taichung, Taiwan, 2002.

[33] J. Usero, F. Rosa, M. Ternero, I. Gracia, "A determination of the sources in the Seville urban aerosol". International Journal of Enviromental Analytical Chemistry, vol 33, pp. 233-244, 1998. 